\title{
A non-perturbative anemometric and flow visualization technique $\left({ }^{+}\right)$
}

\author{
M. Fermigier (*), P. Jenffer (**), J. C. Charmet and E. Guyon $\left({ }^{* *}\right)$ \\ Laboratoire d'Hydrodynamique et de Mécanique Physique, \\ E.S.P.C.I., 10, rue Vauquelin, 75231 Paris Cedex 05, France
}

(Reçu le 10 juillet 1980, accepté le 16 septembre 1980)

\begin{abstract}
Résumé. - Nous présentons une nouvelle technique de visualisation d'un écoulement hydrodynamique utilisant une grille thermique, "écrite " dans le liquide au moyen d'un laser pulsé, dont l'image est ensuite formée, avec une optique classique, par éclairement avec un laser continu. L'expérience donne accès à une nouvelle technique d'anémométrie Doppler qui ne nécessite pas la présence de particules dans le fluide, et à une image instantanée d'un volume fini de l'écoulement.
\end{abstract}

Abstract. - We present a new visualization technique of an hydrodynamic flow using a thermal grid, «written » in the liquid by means of a pulsed laser, and whose image is iubsequently formed, using standard optics, by illumination by a continuous laser.

The experiment gives access to a new Doppler-anemometric technique which does not involve the presence of particles in the fluid and to an instantaneous image of a finite volume of the flow.

The instantaneous visualization and velocity measurement of a finite domain of flow is of considerable interest for unsteady or turbulent velocity field $\mathbf{u}(\mathbf{r}, t)$. Several methods are based on the periodic injection

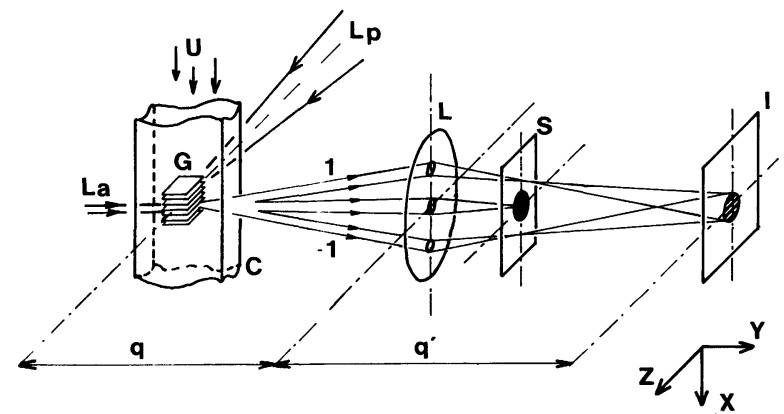

Fig. 1. - In the Poiseuille flow cell (C) a thermal grating $\mathbf{G}$ is formed at initial time $(t=0)$ by the interference of two beams coming from a pulsed Nd YAG laser Lp. The second laser La illuminates this grid and is diffracted (orders $0,1,-1$ ). The Doppler shifted signals $1,-1$ can be used to set an anemometric measurement by standard heterodyne technique. Another possibility is the formation of an image of the grid in the plane I conjugate of $G$ through the lens $\mathrm{L}$. If the laser $\mathrm{La}$ is switched on (by means of an acoustooptic modulator) for a short time period $(t, t+\Delta t)$, the image reflects the integrated effect of the flow field between $t=0$ and $t$.

$\left(^{\dagger}\right)$ Work partially supported by an A.T.P. of C.N.R.S

(*) Thèse de $3^{e}$ Cycle, Université Paris VI (1980).

$\left({ }^{* *}\right)$ Also Université Paris-Sud, 91405 Orsay, France. of lines of lagrangian markers (dye, solid particles, bubbles) in the fluid [1]. The lines distort as they move with the flow, due to the velocity gradients ; a measurement of the integrated displacement and of $\mathbf{u}(\mathbf{r}, t)$ is possible as long as the diffusion has not washed away the lines. The letter discusses a related technique - in which a thermal grid is "written" in a very short time $\left(t_{\mathrm{p}} \sim 10 \mathrm{~ns}\right)$ within a liquid using the pattern formed by the interference of two beams from a pulsed laser Lp [2] (Fig. 1).

In a previous paper [3] we have shown some applications of the forced Rayleigh technique [4] to hydrodynamics : if the thermal grid is "read " by a second laser $\mathrm{La}$ (cw $\mathrm{HeNe} 10 \mathrm{~mW}$ ) incident at right angle with the wave vector of the index grating formed by the thermal grid, the exponential time decay of the intensity of the diffracted peaks gives access to the thermal diffusivity in the flow (Fig. 2a) as in static experiments. Moreover, the rotation and distortion of the grid caused by the velocity gradients are measured through the corresponding changes of the diffraction pattern. The average velocity $\bar{u}$ (in the grid volume) is automatically subtracted by the diffraction process; this is of particular interest for the study of the structure of turbulence which is determined in terms of the gradient components. Moreover, this experiment gives an average information over the size of the laser La. 

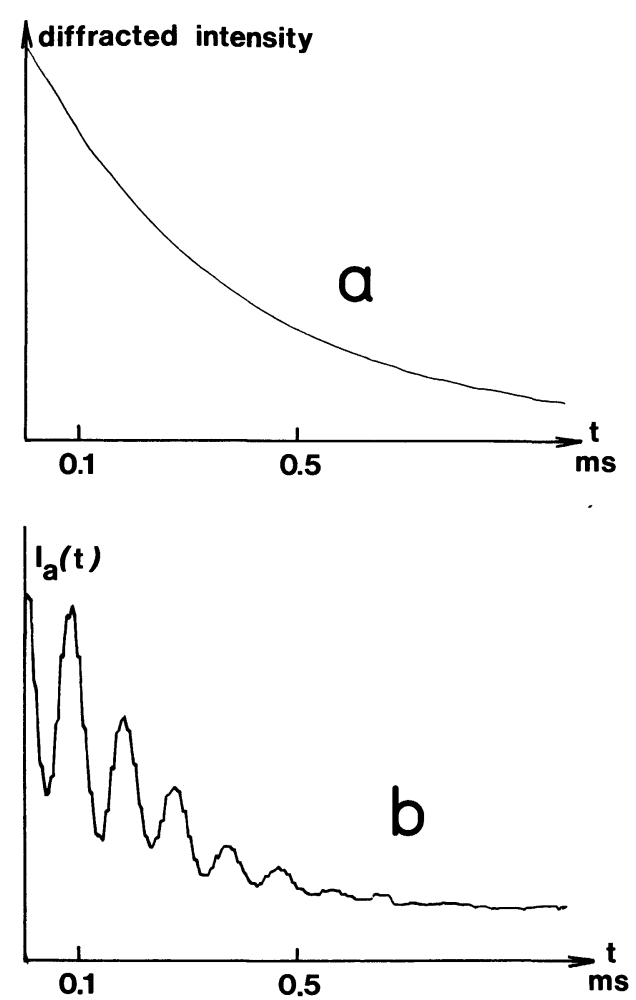

Fig. 2. - a) Classical decay of a forced Rayleigh signal : intensity of the first diffracted order (with an exponential time constant $\tau=p^{2} /\left(8 \pi^{2} \kappa\right)$ where $p$ is the wavelength of the grid, $\kappa$ the thermal diffusivity). $b$ ) The output signal $I_{\mathrm{a}}$ of a photodetector placed in the image of the grid : a periodic modulation is superimposed to the exponential decay with a frequency $2 \Delta v$ proportional to $u$.

The present extension of the technique is quite complementary : i) it permits to observe the thermal grid not only in the Fourier space but also in real space; thus it has spatial resolution; ii) it can give a measure of $\bar{u}$ which, in the forced Rayleigh experiment, was « memorized » as a Doppler shift of the frequency of the diffracted order \pm 1 as $\Delta v= \pm p / \bar{u}$ [1] (where $p=$ period of the thermal grid).

The information in $\bar{u}$ can be extracted by mixing the diffracted beams \pm 1 . This is the principle of the experiment sketched in the right part of figure $1:$ the two beams \pm 1 are recombined to form the image of the grid in a plane I, conjugate of the index grating, through the lens $L$ (the image is magnified by a ratio $\left.m=q^{\prime} / q \sim 10\right)$. In the focal plane of $L$, the screen $S$ acts as an aperture stop in the Fourier space and suppresses the central beam. The strioscopic technique gives an image (amplitude grating) of $100 \%$ contrast. The motion of the image at a velocity $m u(r, t)$ follows that of the object grid.

The photographs (Fig. 3) shows a sequence of two images of a flow. The grid is initially (time $t=0$ ) perpendicular to the plates of a plane Poiseuille flow cell (the flow being uniform along the direction $y$ of the laser La). At a subsequent time $(t=300 \mu \mathrm{s})$, the parabolic velocity profile of the laminar flow is seen from the integrated motion of the grid.

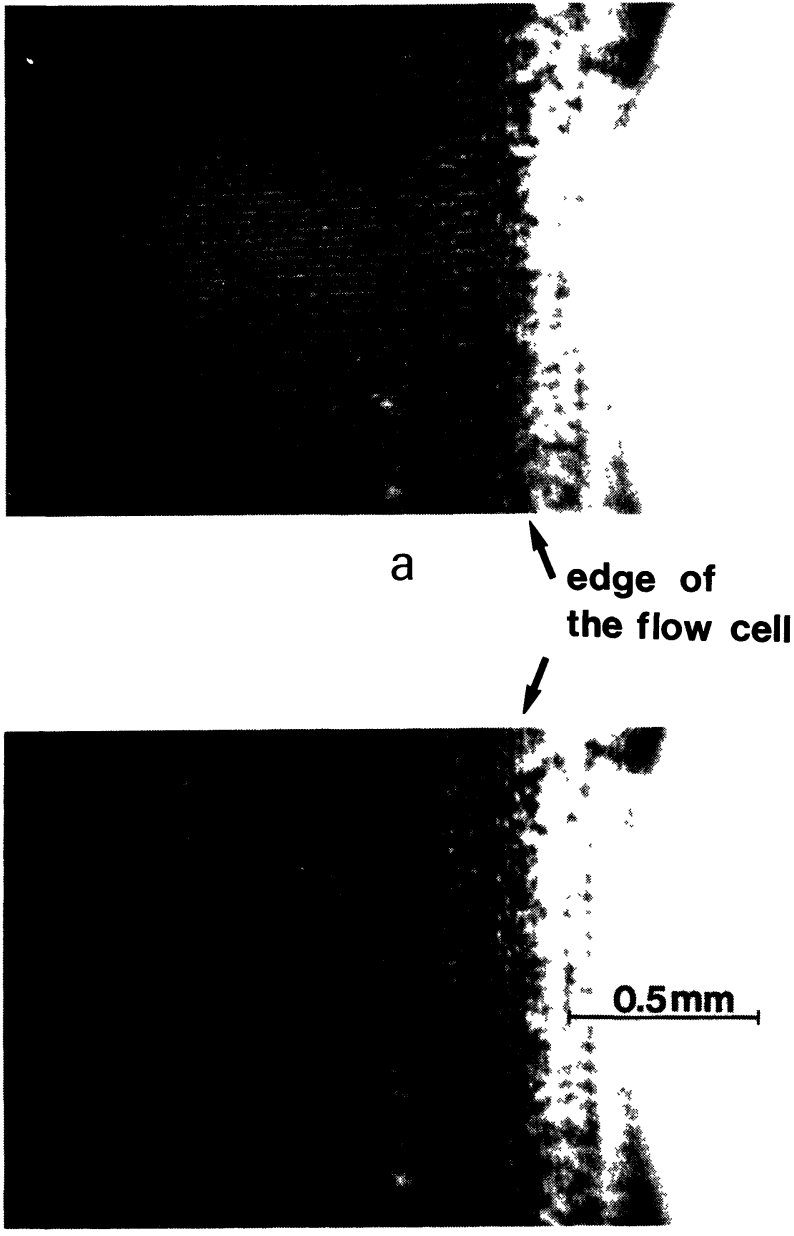

b

Fig. 3. - Photographs of the grid in the image plane $I$ at two different times : a) $t=0$ : right when the grid was formed in the flow cell ; b) $t=300 \mu$ s : grid convected by the Poiseuille flow. We also notice the decay of intensity due to thermal diffusion between the photographs taken with equal exposure time $\Delta t=20 \mu \mathrm{s}$.

One can extend the technique to non uniform Poiseuille flows and suppress the integration along the $y$ direction by replacing the spherical lens used to form the index grating by a cylindrical one. In particular for the visualization of small turbulent structures [5] the thickness of the vertical "ribbon " of pulsed laser light should be kept comparable to the Kolmogorov scale.

The present experiment is limited by the sensitivity of the recording film [6]. In order to avoid fuzzy images, the exposure time has to be kept of the order of $T / 10$, where the period of displacement of the grid is $T \sim 10^{-4} \mathrm{~s}$ for a grating of period $p \simeq 100 \mu \mathrm{m}$ and $u \simeq 1 \mathrm{~m} / \mathrm{s}$. The limit is quite severe for turbulent flows where the attenuation of the grid intensity by turbulent diffusion can be much faster than in laminar flows.

It is also possible to place a small (of extent $<p^{\prime} / 2$ where $p^{\prime}$ is the period of the image grid) photodetector in a prescribed point of the image flow field. The varia- 
tion of intensity with time is given in the figure $2 b$. In addition to exponential diffusive decay, the periodic modulation at a frequency $2 \Delta v$ (given by [1]) is a measure of the local velocity $u(\mathbf{r})$ during the lifetime of the grid. This laser Doppler anemometry technique is complementary to the classical LDA one [7] : in the latter case, particles move within the flow through a fixed grid ; in the present experiment, the grid moves as a whole through the reference line of the La beam. Our experiment is not a true lagrangian one because different parts of the grid pass through the laser beam. However, it offers several advantages : i) it does not require the presence of tracer particles in the flow; ii) it allows the simultaneous measurement of the diffusivity and of one velocity component. Such a joint information can be of interest in particular in the study of two-dimensional turbulent flow fields where it is known that the turbulent mixing can be controled by the large eddy structures [8]; iii) measurement of the velocity in different points of the flow field can be done simultaneously or sequentially by using several small detectors in the image plane I.

Let us finally note that a simpler LDA measurement can be obtained by mixing one of the diffracted beams with unshifted diffused light from the central beam. However in this case we lose the dual information on the map of velocity field obtained by means of the grid image.

\section{References}

[1] Kline, S. J., Flow visualization (Film) in Illustrated experiments in fluid mechanics, National Committee for Fluid Mechanics Films ; MERZ KIRCH, W., Flow Visualization, Chap. 2 (Academic Press) 1974.

[2] Nd YAG laser (Quantel YG 48) frequency doubled $(\lambda=0.53 \mu \mathrm{m})$ with $20 \mathrm{~mJ}$ pulses and $0.5 \mathrm{~Hz}$ repetition rate.

[3] Fermigier, M., Guyon, E., Jenffer, P., Petit, L., Appl. Phys. Lett. 36 (1980) 361.

[4] EICHLeR, H. J., Festkörperprobleme, XXVIII, J. Treusch ed., (Vieweg, Braunschweig) 1978, p. 241.
[5] Consideration of spatial distribution of vorticity at different scales is presently considered as one of the key factors in the understanding of the so-called « homogeneous turbulence $"$.

[6] Kodak Recording 2475 ; it can be processed with a sensitivity of 4000 ASA corresponding to an optical density of 0.1 with an energy density of $10^{-4} \mathrm{erg} / \mathrm{cm}^{2}$.

[7] Durst, F., Melling, A., Whitelaw, J. H., Principles and practice of laser Doppler anemometry (Academic Press) 1976.

[8] Breidenthal, R., AIAA J. 17 (1979) 310. 